\title{
The relationship between emotion regulation capacity, heart rate variability, and quality of life in individuals with alcohol-related brain damage
}

This article was published in the following Dove Press journal:

Psychology Research and Behavior Management

23 August 2016

Number of times this article has been viewed

\author{
Jean-Paul Steinmetz ${ }^{1,2}$ \\ Claus Vögele ${ }^{3,4}$ \\ Christiane Theisen-Flies ${ }^{5}$ \\ Carine Federspiel ${ }^{1,2}$ \\ Stefan Sütterlin ${ }^{6,7}$ \\ 'Department of Research and \\ Development, ZithaSenior, ${ }^{2}$ Centre \\ for Memory and Mobility, ZithaSenior, \\ ${ }^{3}$ Institute for Health and Behaviour, \\ Integrative Research Unit on \\ Social and Individual Development \\ (INSIDE), University of Luxembourg, \\ Luxembourg; ${ }^{4}$ Research Group \\ Health Psychology, University of \\ Leuven, Leuven, Belgium; ${ }^{5} \mathrm{Home} \mathrm{St}$ \\ Joseph, ZithaSenior, Luxembourg; \\ ${ }^{6}$ Department of Psychology, \\ Lillehammer University College, \\ Lillehammer, ${ }^{7}$ Division of Surgery and \\ Clinical Neuroscience, Department \\ of Psychosomatic Medicine, Oslo \\ University Hospital - Rikshospitalet, \\ Oslo, Norway
}

Correspondence: Jean-Paul Steinmetz Department of Research and Development, ZithaSenior, 30, rue Ste Zithe, L-2763 Luxembourg

Tel +352401442060

Fax +352401442952

Email jean-paul.steinmetz@zitha.lu

\begin{abstract}
The reliable measurement of quality of life (QoL) presents a challenge in individuals with alcohol-related brain damage. This study investigated vagally mediated heart rate variability (vmHRV) as a physiological predictor of QoL. Self- and proxy ratings of QoL and dysexecutive symptoms were collected once, while vmHRV was repeatedly assessed over a 3-week period at weekly intervals in a sample of nine alcohol-related brain damaged patients. We provide robustness checks, bootstrapped correlations with confidence intervals, and standard errors for mean scores. We observed low to very low heart rate variability scores in our patients in comparison to norm values found in healthy populations. Proxy ratings of the QoL scale "subjective physical and mental performance" and everyday executive dysfunctions were strongly related to vmHRV. Better proxy-rated QoL and fewer dysexecutive symptoms were observed in those patients with higher vmHRV. Overall, patients showed low parasympathetic activation favoring the occurrence of dysfunctional emotion regulation strategies.
\end{abstract}

Keywords: heart rate variability, emotion regulation, alcohol-related brain damage, quality of life

\section{Introduction}

Healthy individuals without cognitive impairment can adapt flexibly to challenges in their environment, while individuals with cognitive impairment may struggle with behavioral, emotional, and cognitive adaptability. Success in these processes contributes to the person's general well-being and enhances the individual's experience of quality of life (QoL). When evaluating QoL in those without cognitive impairment, clinicians and researchers acknowledge that individuals are the experts of their own QoL. ${ }^{1,2}$ Patients' perceptions of their QoL are, therefore, assessed using self-report measures of QoL, including subjective well-being and/or objective functioning. ${ }^{3}$ Assessing QoL in patients with severe cognitive deficits using such self-report measures, however, poses a challenge due to the nature of their impairment. In patients with alcohol-related brain damage (ARBD), for example, self-report measures of QoL may yield results with questionable reliability and are, therefore, often replaced in clinical practice by expert ratings, that is, proxy measures. Such proxy ratings may provide valuable information of $\mathrm{QoL}$, especially with respect to concrete and observable aspects of QoL. ${ }^{1}$ In using self- and proxy ratings as a source of information, the general phenomenon of judgmental inaccuracies between raters needs to be addressed. Three perspectives on truth in terms of judgmental accuracy have been proposed: consensus, correspondence, and pragmatic accuracy. ${ }^{4}$ Accuracy as consensus refers to the consistency of ratings from at least two different judges. In a recent study 
investigating QoL in ARBDs, we investigated the consensus of QoL evaluations using self- and proxy ratings, the latter provided by health care professionals who were well acquainted with the patients. We observed a significant lack of concordance between both types of QoL ratings, suggesting very low inter-rater consistency. ${ }^{5}$ Interestingly, patients judged their QoL similar to a large healthy normative sample, while health care professionals assessed patients' QoL significantly lower. On the one hand, this finding contradicts previous results in the sense that individuals with alcohol use disorder generally score lower than the general population on a number of QoL-related indices. ${ }^{6}$ On the other hand, the observed gap between self-report and proxy ratings have been reported in previous studies. ${ }^{1,7}$ Nevertheless, a review by Sneeuw et $\mathrm{al}^{8}$ show fair to moderate levels of concordance between patient and physician ratings in general, with physicians' ratings of patients' level of health and functioning tending to be lower than those provided by the patients themselves. The authors conclude from this review that proxy ratings provided by health care providers on several aspects of patients' QoL are reasonably accurate. ${ }^{8}$ However, if patient and proxy ratings are at odds, it is generally accepted that patient ratings should be retained. ${ }^{1}$

It should be noted, however, that studies investigating patient/proxy agreement in QoL include a wide variety of clinical populations. The strength of the reported concordance between patients' and proxy QoL ratings may probably be a function of severity of patients' cognitive impairments. Due to the severity of cognitive impairment experienced by a proportion of individuals with ARBD, patients are no longer able to live independently and, therefore, require long-term, 24-hour care, including basic functions such as personal hygiene. ${ }^{9}$ In addition, most ARBD patients have a history of failed psychiatric rehabilitation and unsuccessful social reintegration, demonstrate a range of cognitive and affective impairments ${ }^{10}$ and considerable levels of anosognosia. ${ }^{5}$ Given their advanced stage of cognitive impairment, patients with ARBD are, therefore, no longer able to lead an independent life. The level of cognitive/affective dysfunction and anosognosia, together with the previously observed repetitive failure of intense therapeutic and rehabilitative measures in these patients prior to their admission casts doubt on the reliability - and hence validity - of self-reported QoL in patients with ARBD. ${ }^{8,11}$ The applicability of the consensus concept of truth ${ }^{4}$ in evaluating QoL in these patient populations may thus be compromised. This is critical, as in addition to complex clinical tests assessing physical health status per se, ratings on QoL and well-being constitute important outcome measures of medical and psychosocial interventions. Improving the reliability of QoL measures in this patient sample, therefore, seems mandatory for both research and clinical practice.

A possible solution may be to refer to the correspondence theory of truth, ${ }^{4}$ defined as the correspondence between a judgment and a more objective criterion. The present study aims at addressing this issue. We investigated vagally mediated heart rate variability (vmHRV), an objectively measurable physiological parameter, as an indicator of emotion regulation capacity. vmHRV has recently been shown to be related to QoL in individuals with compromised intellectual capacities, ${ }^{12}$ making vmHRV a promising candidate to index QoL as a result of an individual's capacity to regulate emotions.

Offline analysis of variability of interbeat intervals in a resting condition allows for the extraction of parameters of prefrontally modulated vagal activation. Prefrontally modulated and vmHRV under resting conditions is considered a marker for regulated emotional responding ${ }^{13}$ and a correlate of prefrontal cortical functions. ${ }^{14}$ Recent research has shown associations between vmHRV and self-reported measures of emotion regulation and QoL in intellectually impaired and visually challenged individuals. ${ }^{12}$ This interpretation with relation to QoL is based on extensive research relating vmHRV to crucial predictors of mental well-being such as successful emotion regulation capacity, ${ }^{13,15,16}$ better self-regulatory skills, ${ }^{17}$ and better top-down modulation of emotional responses due to higher prefrontal cortical inhibitory capacity. ${ }^{14,18,19}$ A lack of prefrontal inhibitory control over subcortical brain regions that are involved in emotional processing gives rise to emotional dysregulation such as emotional instability and perseverative thinking, which are in turn related to impaired life satisfaction and lower QoL. ${ }^{20}$

Low vmHRV is associated with a number of negative health outcomes related to emotional dysregulation such as depression, ${ }^{19,21}$ anxiety, ${ }^{21}$ and stress. ${ }^{22}$ Lower vmHRV has further been related to a range of subclinical risk factors for emotional instability such as less efficient safety learning and extinction of previously learnt stress responses, ${ }^{23}$ increased sensitivity to and dysfunctional cognitive processing of painrelated stimuli, ${ }^{24}$ suboptimal decision-making in risky and emotionally challenging social situations, ${ }^{25,26}$ and emotional instability in everyday life of healthy individuals. ${ }^{15}$

As vmHRV is typically derived from heart rate recordings under resting conditions for a short period of 5 minutes, ${ }^{27}$ it is not affected by confounding influences of participants' cooperation, their motivation, deception, and social 
desirability, which makes it a candidate for an objective biomarker of QoL and well-being in cognitively impaired individuals. Importantly, vmHRV measures obtained at rest are commonly interpreted as trait, but can be affected by state components $^{28}$ that might confound trait-based interpretations (such as trait-QoL). With single measurements, which are common in the vast majority of research on heart rate variability (HRV) and health, the trait component has recently been estimated to be only $49 \%,{ }^{28}$ which gives rise to the assumption that the statistical power of analyses involving measures of HRV assessment is generally overestimated. In the present research, we thus implemented repeated (up to three) measurements and calculated the mean, thereby maximizing the trait-component of our measure in order to compensate for unintended confounding factors by the challenging characteristics of our sample.

Vagally mediated HRV has mostly been used in basic research. In the present study, we aimed to investigate the practicability of vmHRV under the complex conditions found in a normally operating institution. The clinical environment entails practical and methodological limitations that are characteristic for clinical institutions caring for vulnerable and chronic, multimorbid patients. All these characteristics could pose serious limitations to the use of vmHRV as a diagnostic tool in clinical practice. ARBD rarely occurs alone, that is, these patients typically present with a wide range of comorbidities. In the present research, we aimed to investigate a naturalistic sample of patients with ARBD, including the entire range of comorbidities, pharmacological and other treatments, extent of neurological damage, and sociodemographic variables, to best reflect everyday clinical practice. The present sample, therefore, combines a range of patient characteristics that are commonly considered "exclusion criteria" and are not recommended for basic research. Nevertheless, these characteristics are common and constitute a threshold between basic science with high internal but limited external validity and practical use. We are aware that a demonstrated relationship between QoL and vmHRV does not allow for conclusions on direct causal relationships. To demonstrate causality, intervention studies using longitudinal designs are required.

In summary, we investigate the practicability of vmHRV measurement and interpretation for the assessment of wellbeing and QoL in a small naturalistic clinical sample of patients with severe ARBD. More precisely, we conceptualize vmHRV as an objective and trait-related measure of emotion regulation capacity and examine its associations with 1) self-reported and 2) proxy-reported QoL/well-being and the occurrence of dysexecutive symptoms in everyday life, using a correlative within-subject design. Hence, we aim to investigate vmHRVs role as a potential biomarker for QoL and emotion regulation in clinical settings.

\section{Methods \\ Sample}

The initial sample consisted of 18 detoxified patients with chronic alcohol abuse living in a specialized ward providing 24-hour care and support. Of these, four patients withdrew their participation over the course of the investigation (ie, more than one of three HRV assessments missing), and one patient was excluded from the study because of deteriorating health status (benign tumor diagnosis). The remaining sample consisted of 13 patients with a mean age of 58.3 years ( standard deviation $[\mathrm{SD}]=6.9$; range $=45.7-66.5$ ). Given the widespread nature of neurotoxic effects of chronic alcohol abuse, ${ }^{5,10}$ these patients are characterized by significant levels of cognitive and functional impairments requiring 24-hour care and support in a specialized nursing ward. All patients had a history of chronic, heavy alcohol use, repeated relapse, and unsuccessful psychiatric rehabilitation prior to entering the present ward. Due to equipment failures (eg, storage failure, signal loss) an additional four patients were excluded from analysis, resulting in a final sample of nine patients. Table 1 summarizes sample characteristics, including sociodemographic data, medical comorbidities, and current pharmacological treatment. Due to unique characteristics of this particular sample, an age-matched healthy control group was deemed neither necessary nor helpful, as the unambiguous interpretation of group differences in terms of diagnosis would not have been possible due to differences at multiple levels (eg, pharmacotherapy, psychiatric and physical comorbidities, and general lifestyles).

\section{Ethical considerations}

The present study's aims, design, procedure, and publication of anonymous data were reviewed and approved by two independent ethical research boards (Ethics Review Panel, University of Luxembourg [Reference ERP13-021 ALCOQUOL LB/vg]; National Ethical Research Board of Luxembourg [Approval 201310/01]) prior to recruitment and data collection. In addition, the treating physician (ie, an external psychiatrist independent of the present research institutions and without potential conflict of interest) was asked to provide written informed consent on the method, the goal of the study, and the anonymous publication of the results for each participant independently. Furthermore, the general practitioner and legal 
Table I Sociodemographics, medical information, and relevant pharmacological treatment in each patient

\begin{tabular}{|c|c|c|c|c|c|c|c|c|c|}
\hline Characteristics & ARBDI & ARBD2 & ARBD3 & ARBD4 & ARBD5 & ARBD6 & ARBD7 & ARBD8 & ARBD9 \\
\hline \multicolumn{10}{|l|}{ Sociodemographics } \\
\hline Age at time of study (years) & 46.9 & 57.4 & 55.4 & 45.7 & 49.6 & 64.7 & 60.1 & 61.3 & 59.6 \\
\hline $\begin{array}{l}\text { Time living in present ward, at time of study } \\
\text { (months) }\end{array}$ & 3 & 19 & 107 & 4 & 101 & 19 & 106 & 28 & 20 \\
\hline Marital status & Divorced & Married & Single & Divorced & Divorced & Divorced & Divorced & Divorced & Divorced \\
\hline Work status & Invalidity & - & Invalidity & Invalidity & Invalidity & Retired & - & Invalidity & Retired \\
\hline Alcohol abuse $>10$ years & $x$ & - & $x$ & $x$ & $x$ & $x$ & $x$ & $x$ & $x$ \\
\hline History of alcoholism in family & $x$ & - & - & $x$ & - & - & - & $x$ & $x$ \\
\hline \multicolumn{10}{|l|}{ Medical information } \\
\hline ICDI0 diagnosis, primary & FI0.6 & FI0.6 & FI0.6 & FI0.6 & FI0.6 & FI0.6 & FI0.6 & FI0.6 & FI0.6 \\
\hline ICDI0 diagnosis, secondary & K86.0 & $\mathrm{F} 32$ & $\mathrm{~F} 32$ & G62.I & 162.1 & $\mathrm{~F} 32$ & EII & $\mathrm{F} 32$ & $\mathrm{~F} 32$ \\
\hline \multicolumn{10}{|l|}{ CIRS-G } \\
\hline Organ-specific categories endorsed & 4 & 4 & 3 & 4 & 3 & 6 & 4 & 6 & 5 \\
\hline Organ categories with moderate disabilities & 2 & 2 & 1 & 2 & 2 & 3 & 3 & 4 & 2 \\
\hline Overall severity index & 2.0 & 2.0 & 2.0 & 2.0 & 2.3 & 1.8 & 2.3 & 2.0 & 2.2 \\
\hline \multicolumn{10}{|l|}{ Atrophies on MRI } \\
\hline Mammillary bodies & - & $x$ & $x$ & $x$ & - & $x$ & $x$ & $x$ & $x$ \\
\hline Cerebellum & - & - & - & $x$ & - & - & - & - & $x$ \\
\hline Cortex & - & $x$ & $x$ & $x$ & - & $x$ & $x$ & - & - \\
\hline \multicolumn{10}{|l|}{ Pharmacological treatment } \\
\hline Beta-blocker & - & - & - & - & - & - & - & $x$ & $x$ \\
\hline BZD and related substances & - & $x$ & - & $x$ & - & $x$ & - & $x$ & - \\
\hline Neuroleptic & - & - & - & $x$ & $X^{*}$ & - & $x$ & $x$ & - \\
\hline Antidepressant & $x$ & $x$ & $x$ & - & $x$ & $X^{*}$ & - & $x$ & - \\
\hline Thyroid extract & - & - & - & - & - & $x$ & - & - & - \\
\hline
\end{tabular}

Notes: CIRS-G. ${ }^{44}$ Organ-specific categories rated on a Likert-type scale from 0 (no problem) to 4 (extremely severe, organ failure): heart, vascular, hematopoietic, respiratory, eyes/ears/nose/throat/larynx, upper gastrointestinal tract, lower gastrointestinal tract, liver, renal, genitourinary, musculoskeletal-integument, neurological, endocrine-metabolic-breast, and psychiatric illness. A moderate disability is defined by requiring a first-line therapy, overall severity index represents the number of organspecific categories endorsed/total score (unreported here). *If required.

Abbreviations: ARBD, alcohol-related brain damage; BZD, benzodiazepines; CIRS-G, Cumulative Illness Rating Scale for Geriatrics; ICD, International Classification of Diseases; MRI, magnetic resonance imaging; “-”, data not available or condition does not apply; " $X$ ”, condition applies.

guardians of each patient were informed about the study in general and asked to raise objections if applicable. Prior to individual data collection, the health care professional in charge informed the patient about the following procedure including the possibility to withdraw from participation at any time and without any further consequences. (The informed consent process differed from the Declaration of Helsinki of 1975, as revised in 2000 . This is especially the case for $\$ 27, \S 28, \S 29$, and $\S 30$ of the Declaration of Helsinki. By Luxembourg law, legally authorized representatives are not competent in providing written informed consent for the participant to participate in a study [Luxembourg Law of August 11, 1982; see external link \{only available in French language\}: http://www.legilux. public.lu/leg/a/archives/1982/0072/a072.pdf].)

\section{Materials and procedure} Heart rate recording

Measurement of heart rate was performed individually at rest in a separate room with the patient sitting in a comfortable armchair using the heart rate monitor Polar RS800CX
(Polar, Kempele, Finland). ${ }^{29,30}$ Patients were well accustomed to the room as it is located in the ward and is regularly used for relaxation purposes. On the ward, the three health care professionals who were in charge of heart rate measurements were well acquainted with the patients and experienced in conducting relaxation sessions with them. For the purpose of the present study, relaxation sessions were individually performed with one health care professional present in the same room. Patients were accustomed to these kinds of relaxation sessions as they are regularly performed either in a group or individual format. The measurement trial was part of a regularly performed 30 minutes relaxation session, with the patient performing relaxation exercises during the first 15 minutes of the session under the supervision and instruction of the health care professional in charge. For the following 10 minutes, patients were instructed to lean back in the chair, to relax, and to avoid any movements. A 5-minute period of this relaxation phase was used to record heart rate to guarantee maximum relaxation. The last 5 minutes of the 30 minutes session were used to exit the relaxation 
training. Heart rate was recorded three times per participant in identical settings as recommended by Bertsch et al, ${ }^{28}$ with an interval of 1 week between measurements. Each heart rate recording was standardized according to the same protocol, with assessments per participant corresponding to the same period of the day (eg, always in the morning) and with the same health care professional as the preceding assessment.

\section{Self- and proxy ratings}

Self-ratings were collected with the help of a certified clinical psychologist who interviewed each patient on the respective items and who was acquainted with them. We implemented this approach to rule out a possible lack of comprehension given patients' cognitive deficiencies described in Steinmetz and Federspiel. ${ }^{10}$ Proxy ratings differ from self-ratings only by health care professionals being instructed to base their ratings on their personal experiences with and the impressions of the target patient.

\section{QoL ratings}

QoL was assessed using the QoL-profile for chronically ill patients. ${ }^{31}$ This self-rating instrument is a 40 -item generic measure using a 5-point Likert scale to assess the degree of accordance on six different QoL domains: "subjective physical and mental performance" (scale 1), "ability to have pleasure and relaxation" (scale 2), "presence of positive mood" (scale 3), "absence of negative mood" (scale 4), "ability to relate/contact/approach" (scale 5), and "sense of affiliation" (scale 6). The QoL-profile instrument and data are described in a previous publication, ${ }^{5}$ as they were collected in January 2013 and thus $\sim 10$ to 12 months prior to the present study's data collection period (November 2013-January 2014).

\section{Cognitive deficit ratings}

Cognitive impairments were assessed using the Dysexecutive Questionnaire (DEX), a 20-item self- and expert-rating questionnaire taken from the Behavioral Assessment of the Dysexecutive Syndrome battery. ${ }^{32}$ Ratings are made on a 5-point Likert scale (0-4, ranging from "never" to "very often") with a maximum total score of 80 . The DEX assesses the frequency with which observable everyday manifestations of executive dysfunction occur. The DEX was assessed in the same period as vmHRV measurements (data collection period ranging from November 2013 to January 2014).

\section{Data analysis}

Interbeat intervals were retrieved via the software Polar Pro Trainer 5.0 (Polar). All data were screened for measurement errors, these were deleted and substituted by means of cubic spline interpolation, and statistical time and frequency domain measures of vmHRV were obtained. Data processing and statistical analysis followed Task Force recommendations ${ }^{27}$ and was carried out using ARTiiFACT software, ${ }^{33}$ which is based on an error detection algorithm defining individual threshold criteria for erroneously detected interbeat intervals developed by Berntson et al. ${ }^{34}$ The root mean square successive difference (rMSSD) was calculated as a time domain measure. The high frequency component (HF-HRV, 0.15-0.40 Hz) was obtained via fast-Fourier transformation (interpolation rate 4 $\mathrm{Hz}$, window width 256 seconds, window overlap 50\%). Time and frequency domain measures are based on very different statistical approaches, but both are considered to be reliable indicators of vagal activation ${ }^{14,27,28}$ and are usually highly correlated..$^{35-38}$ To maximize the proportion of the trait component and to minimize state-dependent effects on vmHRV, ${ }^{15,28}$ statistical analyses were limited to those participants from whom data could be obtained from at least two measurement sessions. Reported 95\% confidence intervals (CIs) and standard errors (SEs) were estimated using bootstrapping with 1,000 replications. Given the challenging small sample size, significance tests for normalization are not considered sufficiently reliable, therefore both parametric and nonparametric testing procedures were conducted as a conservative and transparent approach to investigate robustness of the findings.

\section{Results}

Table 2 provides descriptive statistics of vmHRV parameters. Compared to available norms in healthy adults, ${ }^{29}$ mean levels of both vmHRV parameters (rMSSD and HF-HRV) in the present patient sample were considerably lower. rMSSD ranged from $3.9(\mathrm{SD}=0.5)$ to $17.5(\mathrm{SD}=5.9)$, with an overall mean of 10.7 ( $\mathrm{SD}=4.5$ ) (bootstrapped: $\mathrm{SE}_{\text {Mean }}=1.4$; CI $[7.9,13.6])$, whereas Nunan et $\mathrm{al}^{39}$ observed an overall mean value of $42(\mathrm{SD}=15)$ ranging between 19 and 75 . For the frequency domain measure HF-HRV, we observed a similar pattern in the present sample ranging from $3.9(\mathrm{SD}=2.1)$ to $163.6(\mathrm{SD}=70.8)$ and an overall mean of $45.5(\mathrm{SD}=29.2)$ (bootstrapped: $\mathrm{SE}_{\text {Mean }}=13.8$; CI [22.5,75.2]), whereas norm values reported by Nunan et $\mathrm{al}^{39}$ are considerably higher, ranging from 82 to 3,630 and a computed cross study overall mean of $657(\mathrm{SD}=777)$.

\section{Self- and proxy ratings}

Results of the QoL measures on a similar but larger patient sample are presented and discussed in Steinmetz et al. ${ }^{5}$ Although these preliminary findings from the present small 
Table 2 QoL and dysexecutive failures as rated by patients and health carers, and heart rate variability results

\begin{tabular}{|c|c|c|c|c|c|c|c|c|c|c|c|c|c|c|c|c|c|c|c|}
\hline & \multicolumn{2}{|c|}{ ARBDI } & \multicolumn{2}{|c|}{ ARBD2 } & \multicolumn{2}{|c|}{ ARBD3 } & \multicolumn{2}{|c|}{ ARBD4 } & \multicolumn{2}{|c|}{ ARBD5 } & \multicolumn{2}{|c|}{ ARBD6 } & \multicolumn{2}{|c|}{ ARBD7 } & \multicolumn{2}{|c|}{ ARBD8 } & \multicolumn{2}{|c|}{ ARBD9 } & \multirow[t]{2}{*}{$P$} \\
\hline & $\mathbf{S}$ & $\mathbf{P}$ & $\mathbf{S}$ & $\mathbf{P}$ & $\mathbf{S}$ & $\mathbf{P}$ & $\mathbf{S}$ & $\mathbf{P}$ & $\mathbf{S}$ & $\mathbf{P}$ & $\mathbf{S}$ & $\mathbf{P}$ & $\mathbf{S}$ & $\mathbf{P}$ & $\mathbf{S}$ & $\mathbf{P}$ & $\mathbf{S}$ & $\mathbf{P}$ & \\
\hline \multicolumn{20}{|l|}{ QoL-profile } \\
\hline $\begin{array}{l}\text { Subjective } \\
\text { physical } \\
\text { and mental } \\
\text { performance } \\
\text { (scale I) }\end{array}$ & - & - & 2.0 & 0.9 & 3.2 & 1.7 & - & - & 2.4 & 2.2 & 2.2 & 1.6 & 2.6 & 2.4 & 1.8 & 1.6 & 3.2 & 2.4 & 0.014 \\
\hline $\begin{array}{l}\text { Ability to have } \\
\text { pleasure and } \\
\text { relaxation } \\
\text { (scale 2) }\end{array}$ & - & - & 2.5 & 1.0 & 3.0 & 2.2 & - & - & 3.0 & 2.6 & 2.7 & 2.4 & 3.1 & 2.9 & 2.4 & 1.7 & 2.4 & 1.6 & 0.006 \\
\hline $\begin{array}{l}\text { Presence of } \\
\text { positive mood } \\
\text { (scale 3) }\end{array}$ & - & - & 1.8 & 0.1 & 3.0 & 1.5 & - & - & 2.8 & 1.8 & 1.6 & 1.7 & 3.2 & 2.4 & 1.8 & 0.7 & 2.2 & 1.3 & 0.004 \\
\hline $\begin{array}{l}\text { Absence of } \\
\text { negative mood } \\
\text { (scale 4) }\end{array}$ & - & - & 2.4 & 0.8 & 2.1 & 2.9 & - & - & 4.0 & 3.3 & 3.6 & 2.9 & 3.6 & 3.4 & 2.7 & 2.2 & 3.1 & 2.2 & 0.098 \\
\hline $\begin{array}{l}\text { Ability to } \\
\text { relate/contact/ } \\
\text { approach } \\
\text { (scale 5) }\end{array}$ & - & - & 1.7 & 0.6 & 3.8 & 1.8 & - & - & 2.8 & 1.6 & 2.3 & 1.8 & 1.7 & 2.7 & 2.5 & 1.2 & 2.7 & 1.7 & 0.059 \\
\hline $\begin{array}{l}\text { Sense of } \\
\text { affiliation } \\
\text { (scale 6) }\end{array}$ & - & - & 3.0 & 1.2 & 3.4 & 2.2 & - & - & 3.4 & 2.6 & 3.4 & 2.5 & 3.4 & 3.1 & 2.2 & 2.5 & 1.4 & 1.4 & 0.050 \\
\hline \multicolumn{20}{|l|}{ DEX } \\
\hline Total score & 16 & 18 & - & - & 22 & 35 & 7 & 41 & 28 & 31 & - & - & 8 & 29 & - & - & - & - & 0.071 \\
\hline \multicolumn{20}{|c|}{ Vagally mediated heart rate variability } \\
\hline $\begin{array}{l}\text { Number of } \\
\text { measurements } \\
\text { Parameters, } \\
\text { mean (SD) }\end{array}$ & \multicolumn{18}{|c|}{$\begin{array}{l}\text { Parameters, } \\
\text { mean (SD) }\end{array}$} & $\mathrm{n} / \mathrm{a}$ \\
\hline $\begin{array}{l}\text { Interbeat } \\
\text { interval }\end{array}$ & \multicolumn{2}{|c|}{$826.4(50.3)$} & \multicolumn{2}{|c|}{$643.8(23.8)$} & \multicolumn{2}{|c|}{$683.6(32.1)$} & \multicolumn{2}{|c|}{$612.3(22.6)$} & \multicolumn{2}{|c|}{$673.5(80.3)$} & \multicolumn{2}{|c|}{794.7 (135.4) } & \multicolumn{2}{|c|}{$794.1(21.3)$} & \multicolumn{2}{|c|}{$730.2(91.5)$} & \multicolumn{2}{|c|}{$729.9(183.2)$} & $\mathrm{n} / \mathrm{a}$ \\
\hline Heart rate & 72.8 & & 93.3( & & 87.9 & & 98.1 & & 89.9( & $0.2)$ & 77.1 & |4.4) & 75.4 & $2.0)$ & 82.8 & 10.4) & 85.3 & & $\mathrm{n} / \mathrm{a}$ \\
\hline rMSSD & 17.5 & & $5.1(2$ & & $8.1(2$ & & $3.9(C$ & & 11.31 & & 11.1 & 1.9) & 15.4 & 1.5) & $1 \mathrm{I} .4$ & & 12.6 & & $\mathrm{n} / \mathrm{a}$ \\
\hline HF-HRV & 163.6 & & $8.6(6$ & & 23.8 & $0.0)$ & $3.9(2$ & & 53.5( & $0.4)$ & 37.1 & 20.9) & 56.9 & 32.1) & 26.3 & 23.4) & 35.5 & & $\mathrm{n} / \mathrm{a}$ \\
\hline
\end{tabular}

Notes: QoL-profile for chronically ill patients. ${ }^{31}$ For scale names, please refer to the text. Higher scores indicate a more positive QoL rating. DEX taken from the BADS. ${ }^{32}$ Higher DEX scores indicate higher ratings on the occurrence of everyday cognitive failures. HF-HRV $(0.15-0.40 \mathrm{~Hz})$ expressed in absolute values $P$ of scale mean differences (dependent $t$-tests, two-tailed) between self- and proxy ratings. Number of measurements, number of vagally mediated heart rate variability assessments available and taken into account per person in the context of the present study.

Abbreviations: ARBD, alcohol-related brain damage; BADS, Behavioral Assessment of the Dysexecutive Syndrome; DEX, Dysexecutive Questionnaire; HF-HRV, high frequency component; n/a, not available; P, proxy rating from acquainted health care professionals; QoL-profile, quality of life profile; rMSSD, root mean square of successive difference; S, self-rating; SD, standard deviation; “-”, data not available or not provided.

sample have limited generalizability, results support these previous findings with QoL ratings from patients being higher on four of the six dimensions than those of the proxies (Table 2). Similarly, proxies rate the frequency of everyday cognitive impairments to be somewhat higher than self-raters suggesting the occurrence of anosognosia in patients. Again, these findings are in line with previous results, ${ }^{5}$ whereas confirmation of the findings is required in other larger samples before firm conclusions on the occurrence of anosognosia in patients with ARBD can be drawn.

\section{Relationships of vmHRV with QoL and DEX}

Bootstrapped correlation analyses between rMSSD and self- and proxy-rated QoL revealed an interesting pattern of relationships. Linear relationships that are discussed were investigated using Pearson's correlation coefficient $r$. When using Spearman's rank order correlation coefficient (rho), the pattern of correlations was similar to Pearson's $r$, except for the QoL-profile scale 4 (absence of negative mood) (details are presented in Table 3). 


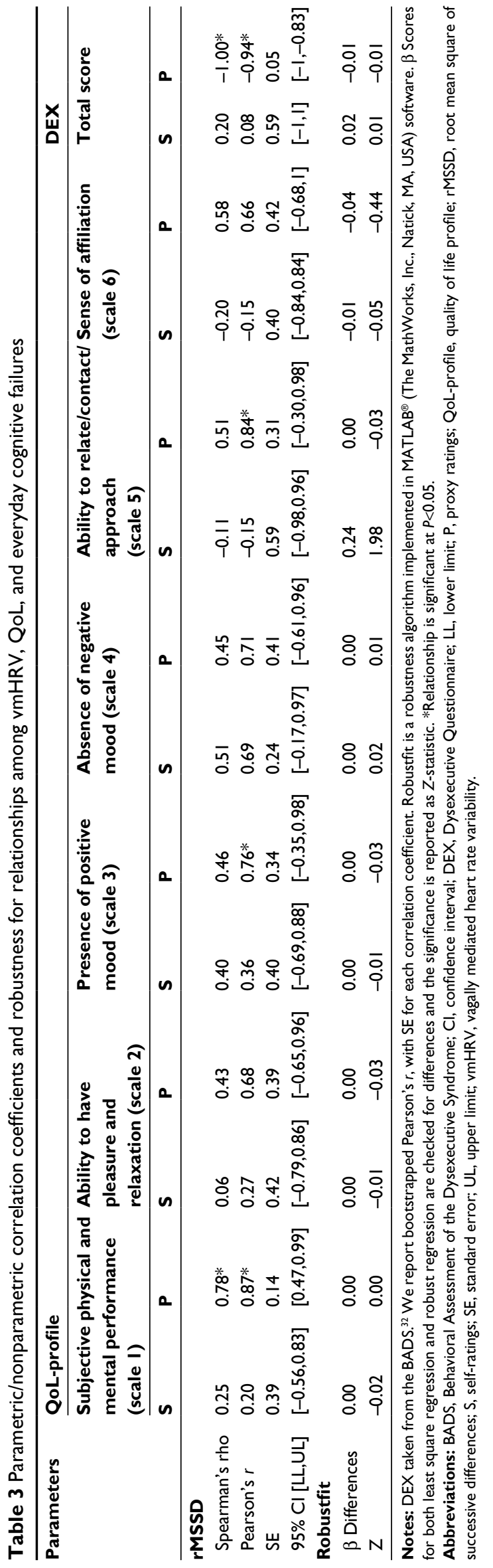

Small- to moderate-sized correlations (total mean $r=|0.30|)$ were observed between MSSSD and self-rated QoL (with one exception, scale 4 in Table 3), whereas correlations between rMSSD and proxy-rated QoL were considerably higher (total mean $r=|0.75|$ ) (Table 3 ). When inspecting corresponding SEs and 95\% CIs of these correlations, only proxy-rated QoL-profile scale "subjective physical and mental performance" (scale 1) remained valid with a CI ranging from 0.47 to 0.99 (Table 3 ). The remaining correlations between self- and proxy-rated QoL with rMSSD had large SEs and hence large 95\% CIs and thus warrant no further interpretation. Considering correlations between self-rated everyday cognitive failures (DEX total score), a weak relationship was observed with $\mathrm{rMSSD}(r=-0.08$, rho $=0.20$ ), whereas a nearly perfect negative relationship with rMSSD was observed for the proxy rating ( $r=-0.94)$, with $95 \%$ CIs ranging from -1.00 to -0.84 (Table 3 ). Robustness of correlations was checked using the robustfit algorithm implemented in MATLAB ${ }^{\circledR}$ software (The MathWorks, Inc., Natick, MA, USA). The robustfit function computes the difference between ordinary least square regressions and robust regressions, yielding nonsignificant $\beta$ differences between least square and robust regressions $(-1.96<\mathrm{Z}>+1.96)$, with one exception (self-rated QoL scale 5 with rMSSD, Figure 1). Nonsignificant $\beta$ differences indicate that linear relationships are relatively robust with no or only minor influences from extreme scores (ie, outliers) on one or the other variable.

\section{Discussion}

The purpose of the present study was to investigate the usefulness of vmHRV as a correlate of well-being and QoL in a clinical sample of severely deteriorated ARBD individuals. We related the patient's emotion regulation capacity with self- and proxy-rated QoL/well-being. Additionally, we investigated possible relationships of vmHRV with self- and proxy-reported executive failures.

In general, we observed low to very low HRV scores compared to healthy adults. ${ }^{39}$ Although preliminary, this suggests a remarkably low tonic parasympathetic activation that might indicate diminished prefrontal inhibitory control in these patients. Limited inhibitory control may favor the occurrence of dysfunctional psychological adaptation to internal or external input and hence, emotional dysregulation in daily life, ${ }^{15}$ reduced general self-regulatory abilities, ${ }^{17,19}$ and overall reduced QoL. ${ }^{12}$ Previously, low vmHRV has been associated with numerous negative health outcomes related to emotional dysregulation. Our sample is characterized by a relatively high prevalence of psychiatric 
A

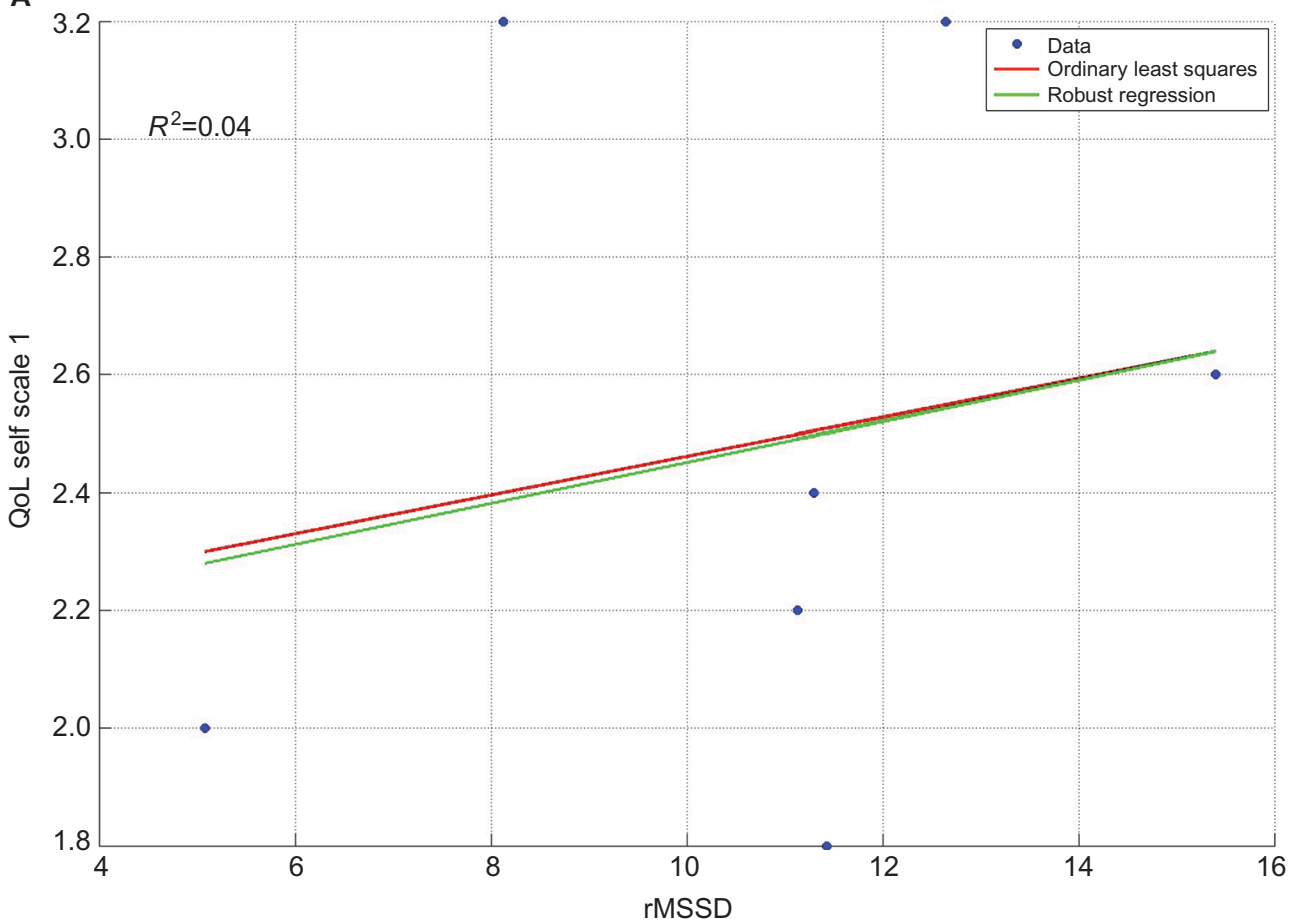

B

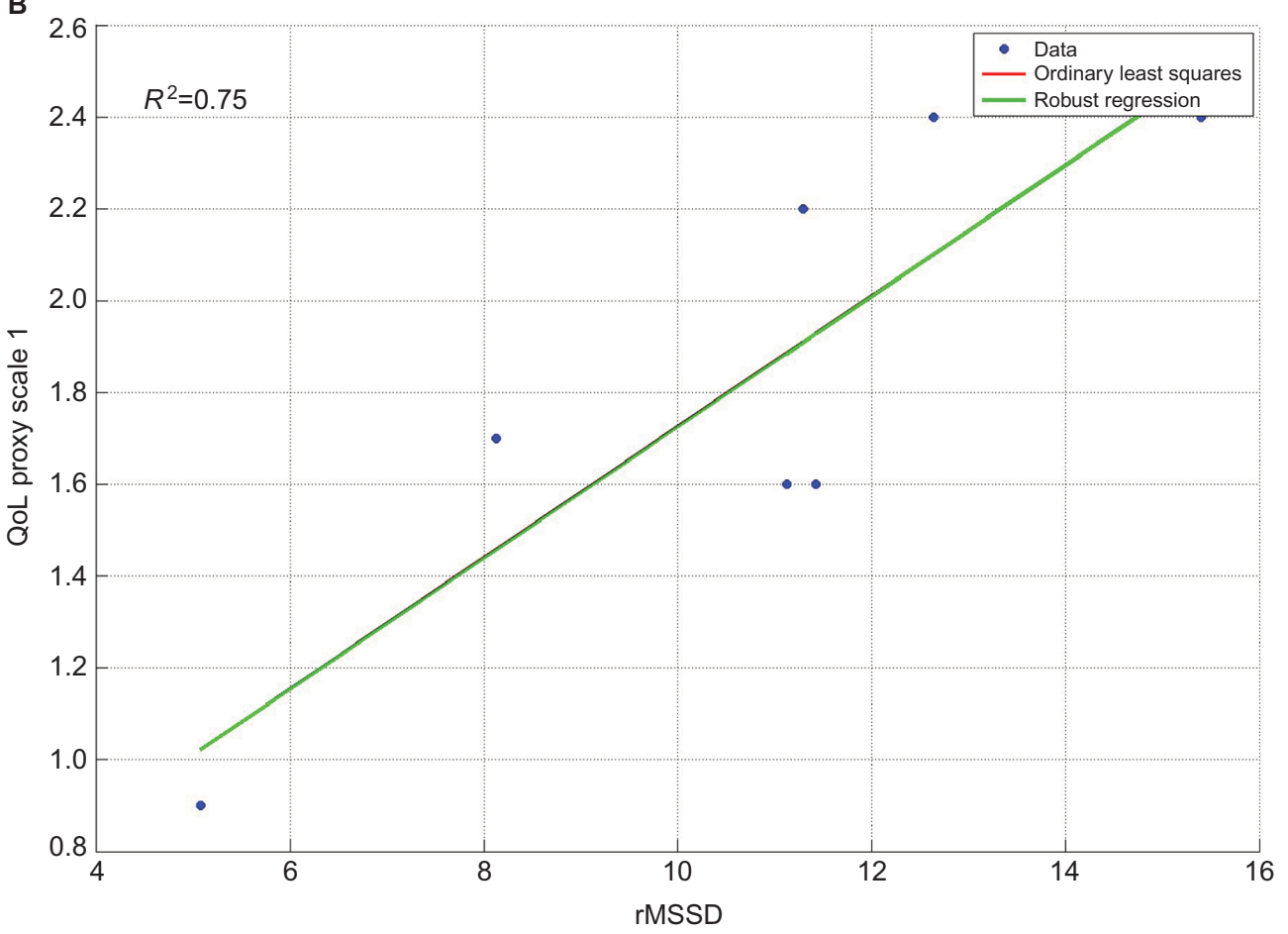

Figure I (Continued) 

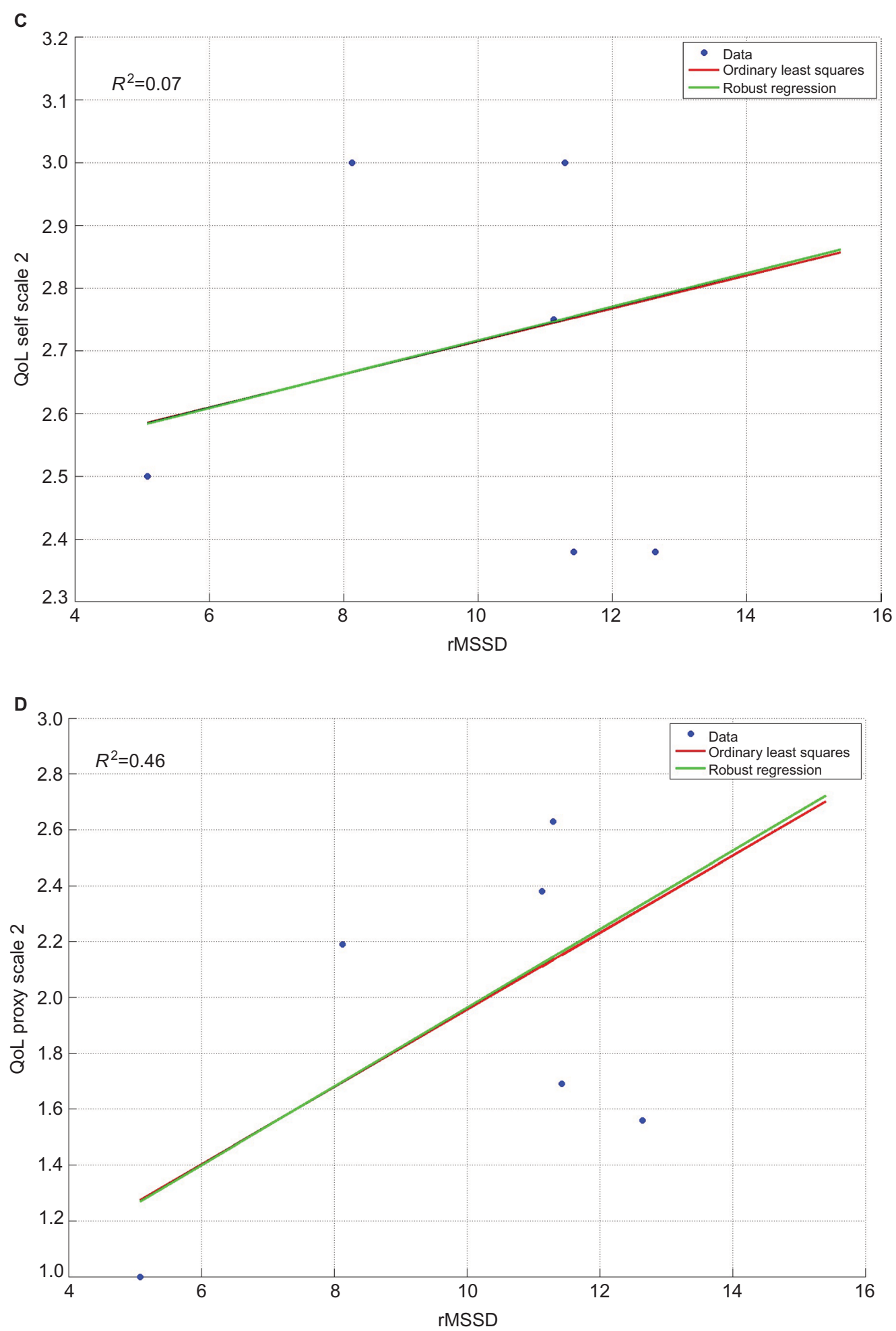

Figure I (Continued) 

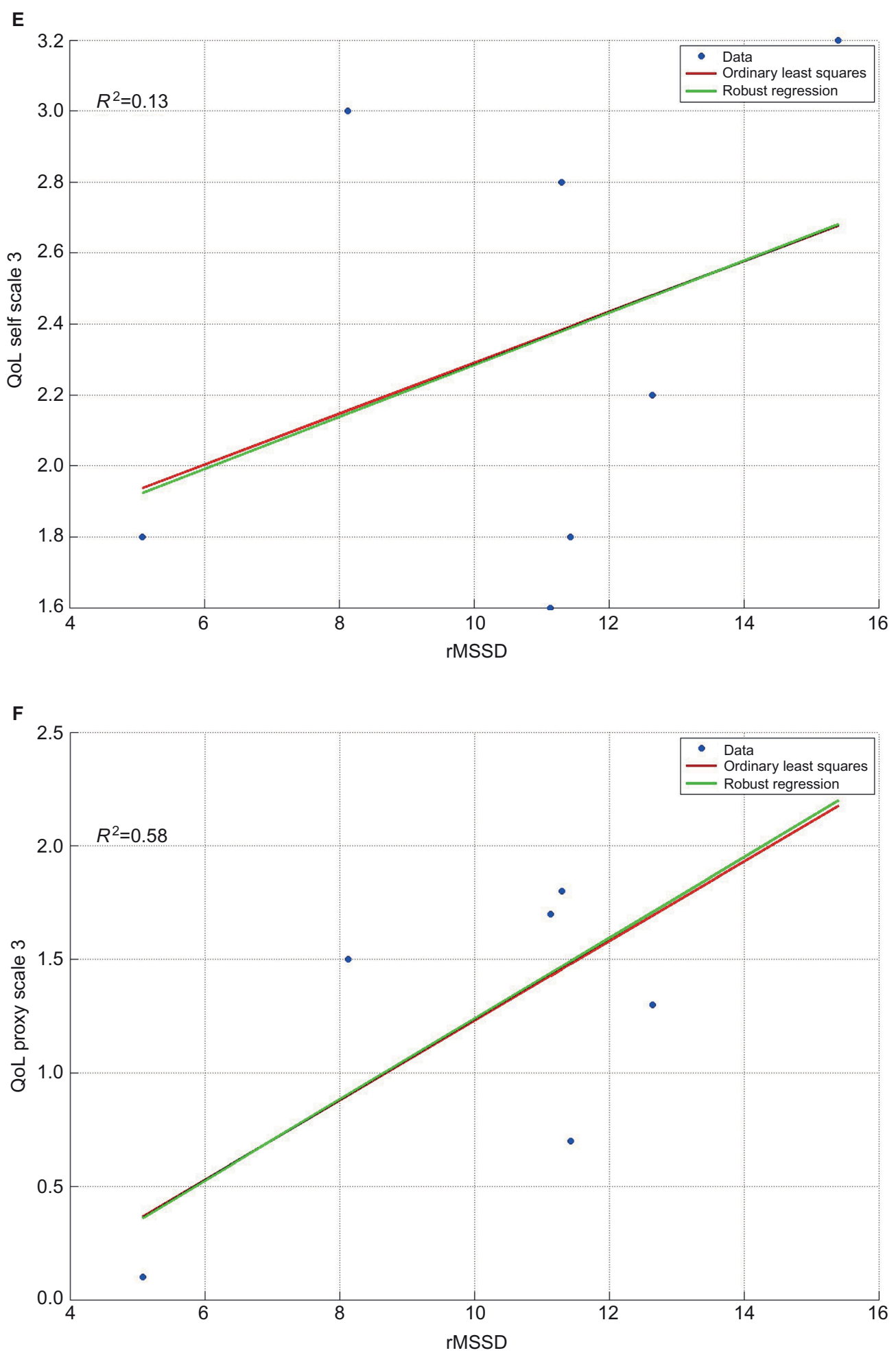

Figure I (Continued) 


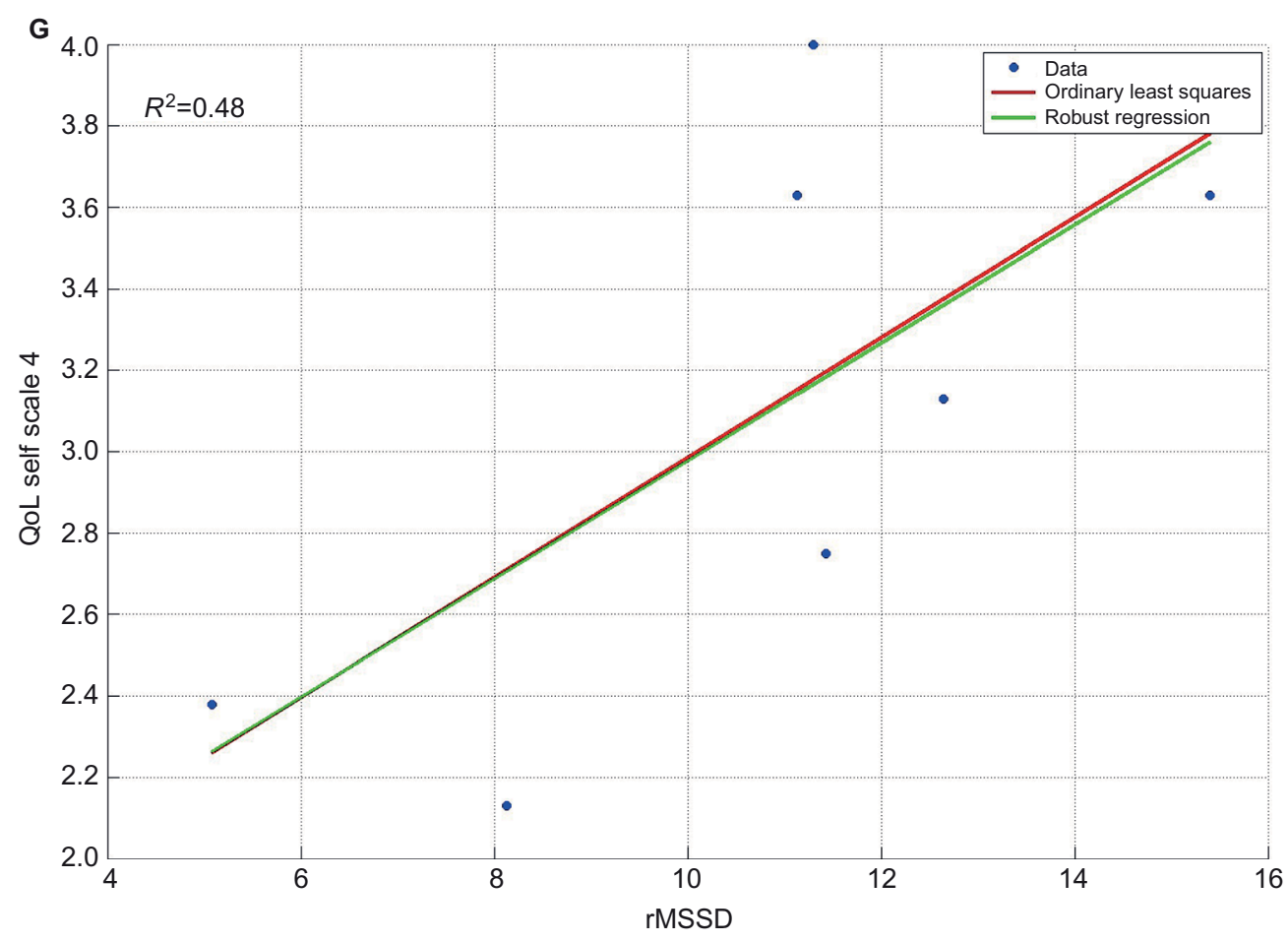

H

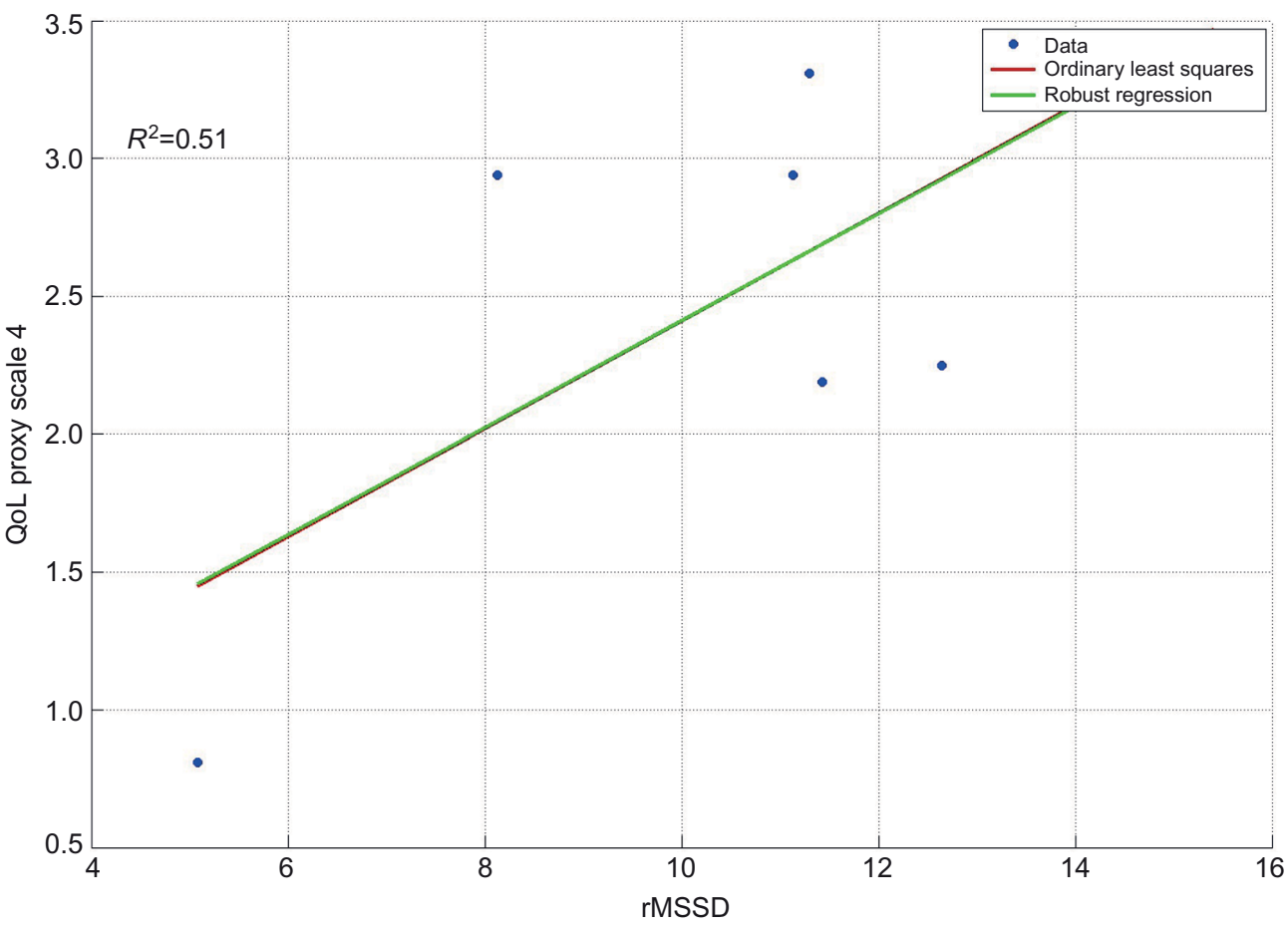

Figure I (Continued) 

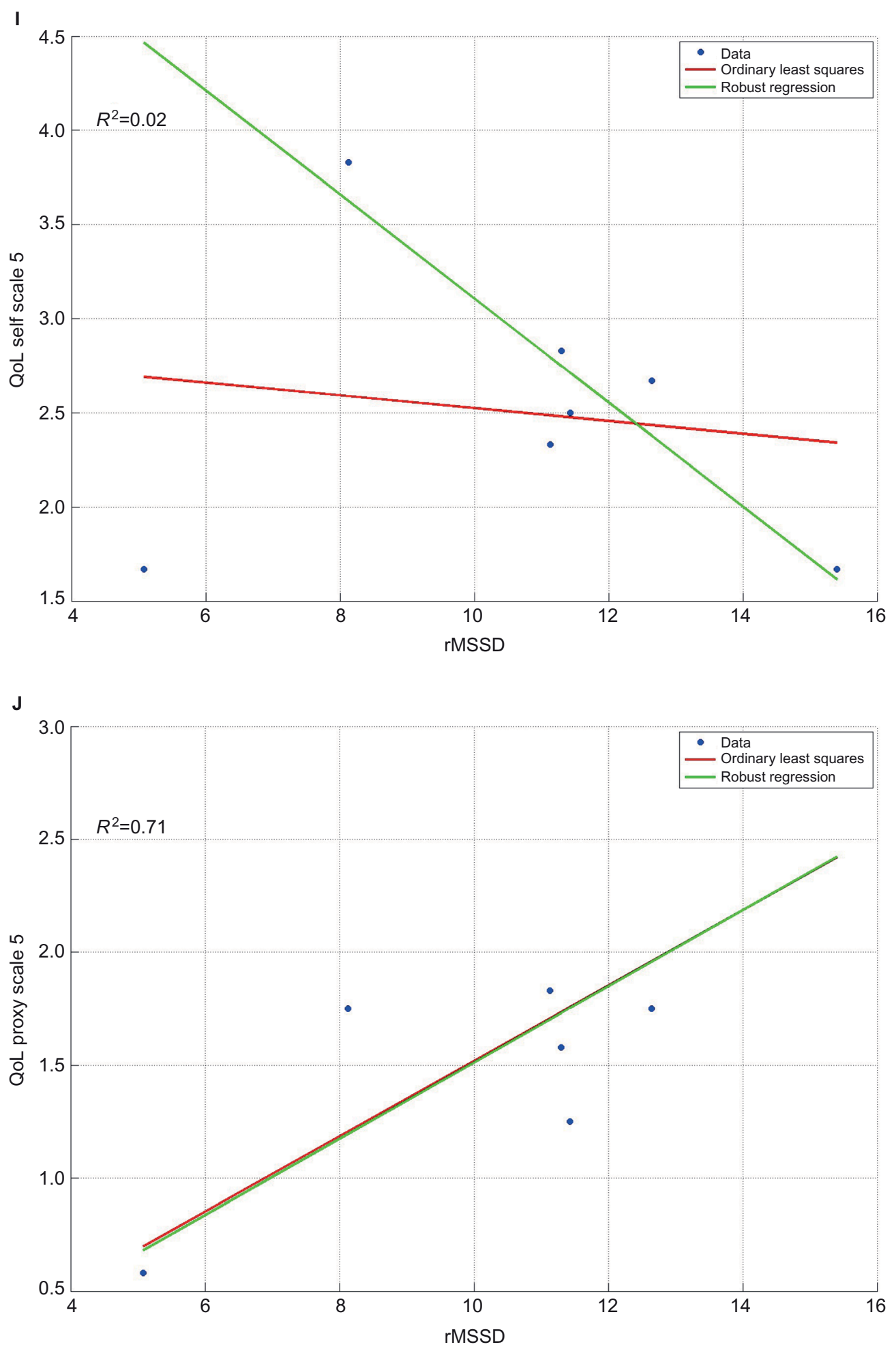

Figure I (Continued) 

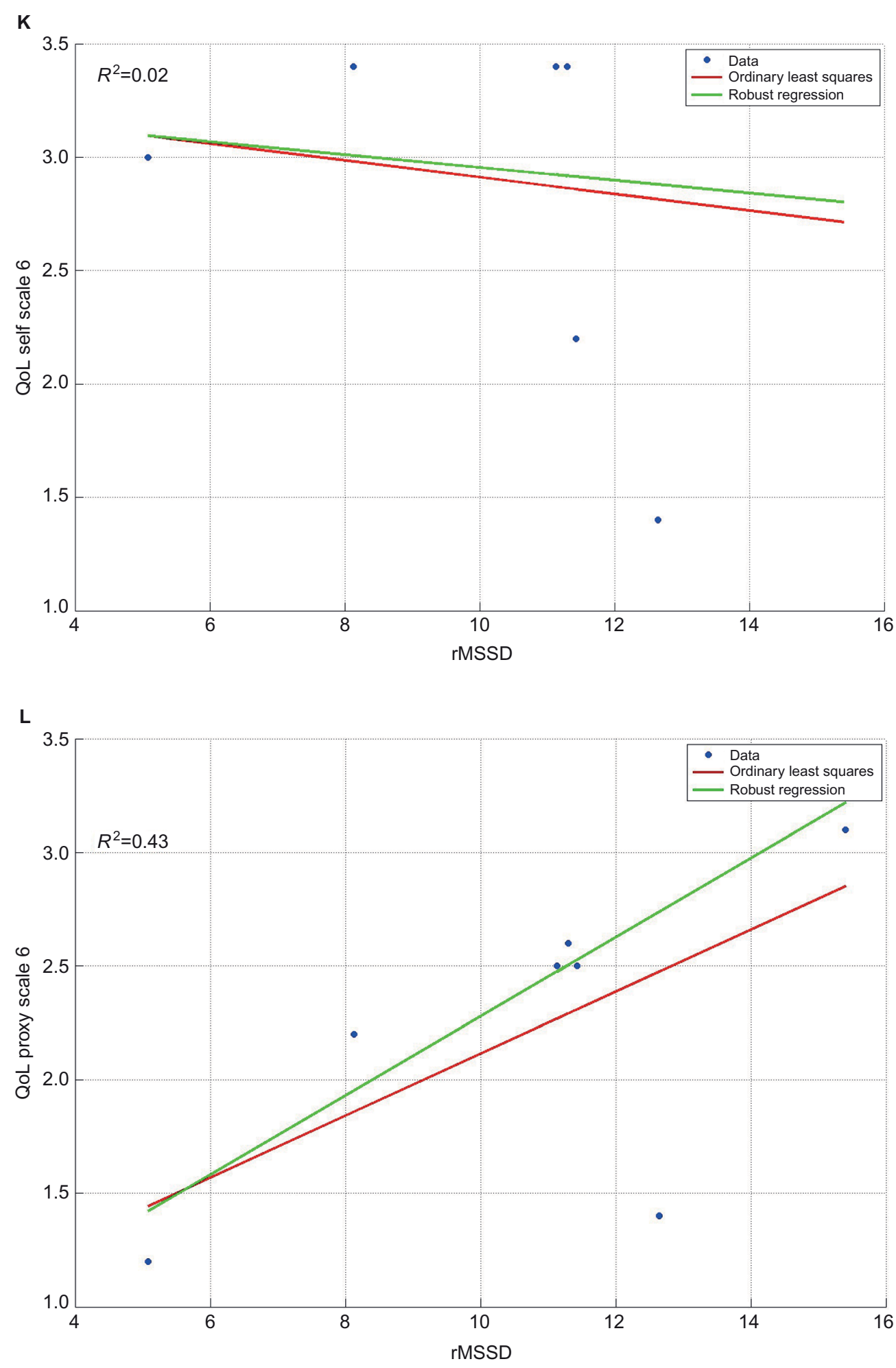

Figure I (Continued) 

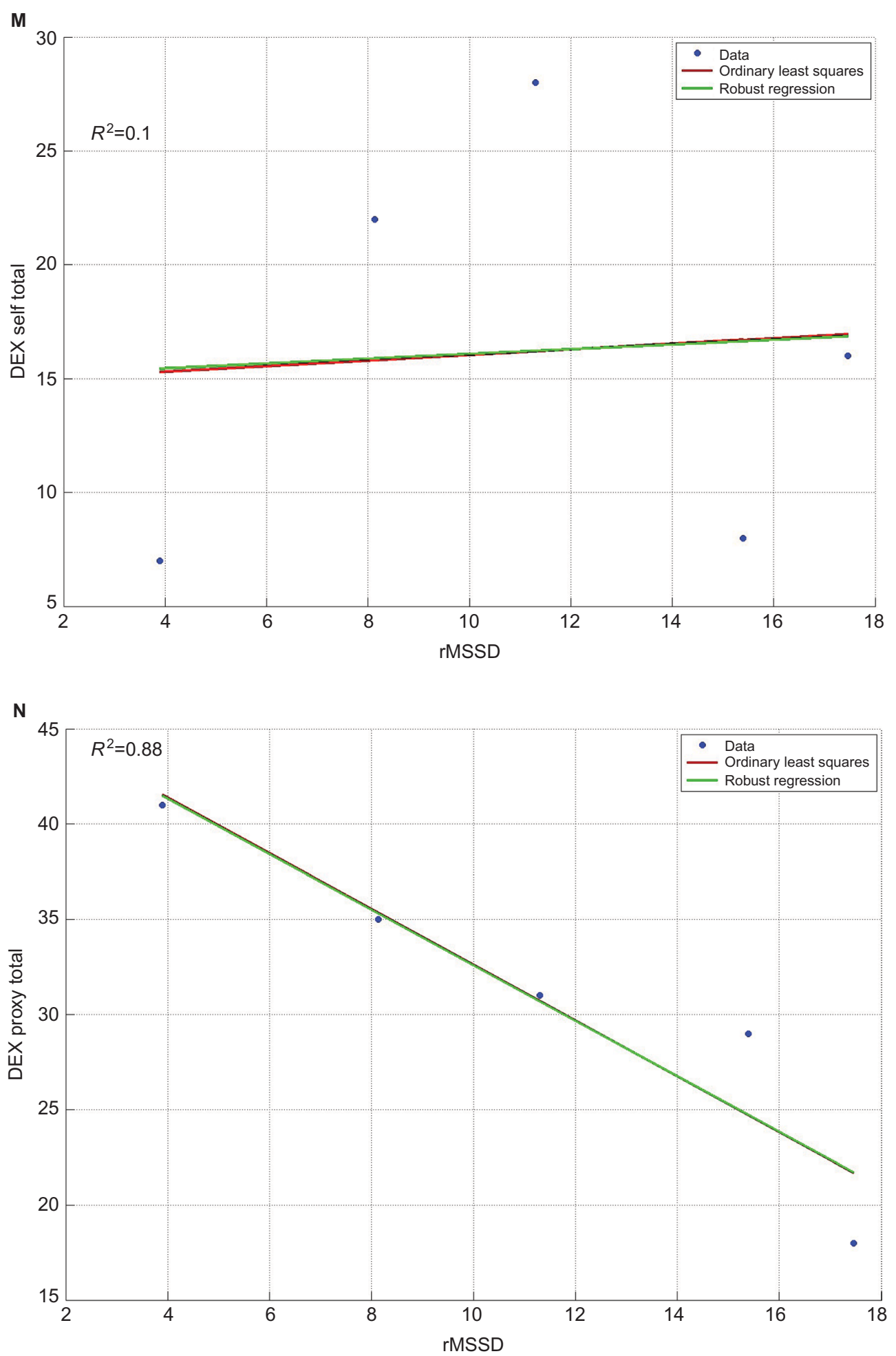

Figure I Comparison of ordinary least square regressions and robust regressions for self- and proxy ratings on the six QoL-profile scales (A-L) and the occurrence of everyday executive dysfunctions ( $\mathbf{M}$ and $\mathbf{N}$ ).

Notes: QoL-profile for chronically ill patients. ${ }^{31}$ For scale names, please refer to the text. Higher scores indicate a more positive QoL rating. DEX taken from the BADS. ${ }^{32}$ Higher DEX scores indicate higher ratings on the occurrence of everyday cognitive dysfunctions. The red line represents the ordinary least squares regression line. The green line represents the robust regression computed by the robustfit algorithm implemented in MATLAB ${ }^{\circledR}$ software (The MathWorks, Inc., Natick, MA, USA).

Abbreviations: BADS, Behavioral Assessment of the Dysexecutive Syndrome; DEX, Dysexecutive Questionnaire; rMSSD, root mean square successive difference; QoLprofile, quality of life profile. 
illnesses (eg, depressions, anxiety), with seven out of nine individuals $(78 \%)$ suffering from symptoms requiring first-line treatment (unpublished data using the CIRS-G instrument). Although causal relationships should not be inferred from the present data, the present results may well have clinical implications. HRV biofeedback interventions have been shown to improve symptoms in patients with major depression and anxiety disorders related to emotional and behavioral dysregulation. ${ }^{40}$

The present findings support the notion that judgments of patient's QoL obtained from acquainted health care professionals are a valuable and reasonably accurate source of information. ${ }^{8}$ Our findings further demonstrate that patients' HRV is related to QoL ratings obtained from acquainted health care professionals. HRV was observed to be higher in patients that were rated as experiencing better QoL and demonstrated fewer dysexecutive symptoms. In contrast, we observed mostly weak associations between self-rated QoL and patients' vmHRV, suggesting a nil relationship between both variables. Hence, by considering only patient ratings in the present sample, important health-related information on quality of care and QoL of the patient would have been missed.

As pointed out previously, the present sample is atypical for research studies investigating vmHRV and its relationship to other constructs. We investigated a small clinical sample with a range of comorbidities requiring therapy and, therefore, compromising internal validity of the study. We cannot exclude that the observed deficits or relationships are independent of other causes (eg, head injury, dementia unrelated to alcoholism, liver diseases, etc). Importantly and although a large body of research discusses the psychological correlates of vmHRV in healthy and clinical samples, many studies suffer from limited ecological validity due to restrictive inclusion and exclusion criteria. This study put previous findings into practice and investigated QoL as a previously reported correlate of QoL in a "naturalistic" setting in a challenging small and highly comorbid sample. A range of measures have been applied to test for robustness of the results. First, data were qualitatively inspected and visualized to check for possible outliers. In this context, robustness of the reported linear relationships was checked and beta weight differences are reported. The reported patterns of relationships between vmHRV and all six QoL dimensions are consistent. That is, proxy ratings are positively and strongly related to vmHRV, whereas mostly weak or nil relationships are observed between self-ratings and vmHRV. In addition, vmHRV is strongly and consistently related to the proxy-reported occurrence of executive dysfunctions (DEX total score), whereas again, weak/nil relationships are observed between the self-reported executive dysfunctions and vmHRV. This pattern of relationships is reproduced when inspecting correlations between the three underlying DEX factors: cognition, behavior, and emotion. ${ }^{32,41-43}$ Second, we provide bootstrapped 95\% CIs and SEs for the computed central tendencies and correlations. Third, the present study maximizes the trait-component of baseline vmHRV measures by averaging over more than one assessment. This contributes to the stability and internal validity of the present findings. ${ }^{28}$ In the present study, no significant findings based on single measurements were found (unreported here).

To the best of our knowledge, the present study is the first to investigate direct relationships between emotion regulation capacity, the occurrence of everyday executive dysfunctions, and QoL in a sample of chronic patients with severe multimorbid conditions. The present preliminary results suggest that vmHRV demonstrates construct validity, given the relative stability of correlations investigated. While internal validity of the present study is limited, its external validity would seem to be adequate. More precisely, external validity is important as it demonstrates the applicability of the present research design and approach to a highly complex multimorbid clinical sample. We were able to perform accurate and repeated assessments of vmHRV in a complex clinical context with a wide variety of intervening confounding factors. Thus, our paper contributes to the applicability of vmHRV by stressing the necessity of multiple measurements to ensure trait characteristics of HRV. Pending further research and replication in larger samples, HRV could be useful in situations where expert ratings are unclear (eg, disagreement within a team, high degree of staff fluctuation in transition periods). First studies on applying vmHRV assessments to clinical samples have been published. ${ }^{12}$

\section{Conclusion}

The present study demonstrates that individuals with ARBD have low to very low HRV scores compared to healthy adults and that patients' HRV is related to QoL ratings obtained from acquainted health care professionals. We demonstrated the construct validity of vmHRV, given the relative stability of the investigated associations. We performed accurate and repeated assessments of vmHRV in a complex clinical context with many intervening factors. We thus see the contribution of this research in the fact that we put a well-known and as such well-established psychophysiological measure to the test 
under challenging contextual situations in a natural setting (low sample size, heterogeneous sample with multiple comorbidities, and intellectual impairment). Hence, we conclude that demonstrating the practical use of a tool and applicability of its measures in many diverse clinical settings is a crucial and necessary step for this promising method to slowly move from the laboratory to the clinical field.

\section{Acknowledgments}

The authors are grateful to the patients, their families, and nursing staff for their participation and support during the study. They would like to thank Dr Tobias Kaufmann, Institute of Clinical Medicine at the University of Oslo, for his assistance during data analysis. This work was supported by continuous funding from the Foundation Ste Zithe Luxembourg which is gratefully acknowledged. The supporting source had no involvement in the study design, data collection, data analysis, interpretation of the data, in the writing of report, or in the decision to submit the manuscript for publication. Parts of the study were presented as a poster at the 22nd Nordic Congress of Gerontology in Gothenburg, Sweden, May 26-28, 2014.

\section{Author contributions}

J-PS, CV, CT-F, CF, and SS conceived the design of the study. J-PS and SS conducted the literature search. J-PS and SS were responsible for the first draft of the manuscript. CT-F obtained the study data. J-PS and CF acquired funding for the study. J-PS and SS carried out the statistical analyses. J-PS and SS contributed to the interpretation of the study findings. J-PS, $\mathrm{CV}, \mathrm{CT}-\mathrm{F}, \mathrm{CF}$, and SS contributed to the critical revision of the paper and approved the final manuscript.

\section{Disclosure}

The authors report no conflicts of interest in this work.

\section{References}

1. Addington-Hall J, Kalra L. Who should measure quality of life? Br Med J. 2001;322(7299):1417-1420.

2. Slevin ML, Plant H, Lynch D, Drinkwater J, Gregory WM. Who should measure quality of life, the doctor or the patient? Br J Cancer. 1988;57(1):109-112.

3. Muldoon MF, Barger SD, Flory JD, Manuck SB. What are quality of life measurements measuring? Br Med J. 1998;316(7130):542-544.

4. Kruglanski AW. The psychology of being "right": the problem of accuracy in social perception and cognition. Psychol Bull. 1989;106(3):395-409.

5. Steinmetz J-P, Theisen-Flies C, Federspiel C. Views on quality of life differ between alcohol related brain damaged individuals and their healthcare professionals. Appl Res Qual Life. 2016;11(1):239-251.

6. Foster JH, Peters TJ, Marshall EJ. Quality of life measures and outcome in alcohol-dependent men and women. Alcohol. 2000;22(1):45-52.

7. Sprangers MA, Aaronson NK. The role of health care providers and significant others in evaluating the quality of life of patients with chronic disease: a review. J Clin Epidemiol. 1992;45(7):743-760.
8. Sneeuw KCA, Sprangers MA, Aaronson NK. The role of health care providers and significant others in evaluating the quality of life of patients with chronic disease. J Clin Epidemiol. 2002;55(11):1130-1143.

9. National Institute for Health and Clinical Excellence (NICE). AlcoholUse Disorders: Diagnosis, Assessment and Management of Harmful Drinking and Alcohol Dependence. London, UK: National Institute for Health and Clinical Excellence; 2011.

10. Steinmetz J-P, Federspiel C. Alcohol-related cognitive and affective impairments in a sample of long-term care residents. GeroPsych. 2012;25(2):83-95.

11. Murrell R. Quality of life and neurological illness: a review of the literature. Neuropsychol Rev. 1999;9(4):209-229.

12. Meule A, Fath K, Real RG, Sütterlin S, Vögele C, Kübler A. Quality of life, emotion regulation, and heart rate variability in individuals with intellectual disabilities and concomitant impaired vision. Psychol WellTheory Res Pract. 2013;3(1):1.

13. Appelhans BM, Luecken LJ. Heart rate variability as an index of regulated emotional responding. Rev Gen Psychol. 2006;10(3):229-240.

14. Thayer JF, Åhs F, Fredrikson M, Sollers JJ 3rd, Wager TD. A metaanalysis of heart rate variability and neuroimaging studies: implications for heart rate variability as a marker of stress and health. Neurosci Biobehav Rev. 2012;36(2):747-756.

15. Koval P, Ogrinz B, Kuppens P, Van den Bergh O, Tuerlinckx F, Sütterlin S. Affective instability in daily life is predicted by resting heart rate variability. PLoS One. 2013;8(11):e81536.

16. Thayer JF, Siegle GJ. Neurovisceral integration in cardiac and emotional regulation. IEEE Eng Med Biol Mag. 2002;21(4):24-29.

17. Segerstrom SC, Nes LS. Heart rate variability reflects self-regulatory strength, effort, and fatigue. Psychol Sci. 2007;18(3):275-281.

18. Thayer JF, Hansen AL, Saus-Rose E, Johnsen BH. Heart rate variability, prefrontal neural function, and cognitive performance: the neurovisceral integration perspective on self-regulation, adaptation, and health. Ann Behav Med. 2009;37(2):141-153.

19. Thayer JF, Lane RD. Claude Bernard and the heart-brain connection: further elaboration of a model of neurovisceral integration. Neurosci Biobehav Rev. 2009;33(2):81-88.

20. Sütterlin S, Paap MCS, Babic S, Kübler A, Vögele C. Rumination and age: some things get better. J Aging Res. 2012;2012:267327.

21. Kemp AH, Quintana DS, Felmingham KL, Matthews S, Jelinek HF. Depression, comorbid anxiety disorders, and heart rate variability in physically healthy, unmedicated patients: implications for cardiovascular risk. PloS One. 2012;7(2):e30777.

22. Jarczok MN, Jarczok M, Mauss D, Koenig J, Li J, Herr RM, Thayer JF. Autonomic nervous system activity and workplace stressors - a systematic review. Neurosci Biobehav Rev. 2013;37(8):1810-1823.

23. Pappens M, Schroijen M, Sütterlin S, Smets E, Van den Bergh O, Thayer JF, Van Diest I. Resting heart rate variability predicts safety learning and fear extinction in an interoceptive fear conditioning paradigm. PLoS One. 2014;9(9):e105054.

24. Scheuren R, Sütterlin S, Anton F. Rumination and interoceptive accuracy predict the occurrence of the thermal grill illusion of pain. $B M C$ Psychol. 2014;2(1):22.

25. Sütterlin S, Herbert C, Schmitt M, Kübler A, Vögele C. Frames, decisions, and cardiac-autonomic control. Soc Neurosci. 2011;6(2):169-177.

26. Sütterlin S, Herbert C, Schmitt M, Kübler A, Vögele C. Overcoming selfishness: reciprocity, inhibition, and cardiac-autonomic control in the ultimatum game. Front Psychol. 2011;2:173.

27. [No authors listed]. Task Force of the European Society of Cardiology and the North American Society of Pacing and Electrophysiology. Heart rate variability. Standards of measurement, physiological interpretation, and clinical use. Eur Heart J. 1996;17(3):354-381.

28. Bertsch K, Hagemann D, Naumann E, Schächinger H, Schulz A. Stability of heart rate variability indices reflecting parasympathetic activity. Psychophysiology. 2012;49(5):672-682.

29. Nunan D, Jakovljevic DG, Donovan G, Hodges LD, Sandercock GRH, Brodie DA. Levels of agreement for RR intervals and short-term heart rate variability obtained from the Polar S810 and an alternative system. Eur J Appl Physiol. 2008;103(5):529-537. 
30. Weippert M, Kumar M, Kreuzfeld S, Arndt D, Rieger A, Stoll R. Comparison of three mobile devices for measuring R-R intervals and heart rate variability: Polar S810i, Suunto t6 and an ambulatory ECG system. Eur J Appl Physiol. 2010;109(4):779-786.

31. Laubach W, Schröder C, Siegrist J, Brähler E. Normierung der Skalen "Profil der Lebensqualität Chronisch Kranker" an einer repräsentativen deutschen Stichprobe [Standardization of the scale "Quality of life profile for Chronically Ill Individuals" in a representative German sample]. Z Für Differ Diagn Psychol. 2001;22(2):100-110.

32. Wilson BA, Alderman N, Burgess PW, Emslie H, Hodges JJ. Behavioural Assessment of the Dysexecutive Syndrome (BADS). Bury St Edmunds (UK): Thames Valley Test Company; 1996.

33. Kaufmann T, Sütterlin S, Schulz SM, Vögele C. ARTiiFACT: a tool for heart rate artifact processing and heart rate variability analysis. Behav Res Methods. 2011;43(4):1161-1170.

34. Berntson GG, Quigley KS, Jang JF, Boysen ST. An approach to artifact identification: application to heart period data. Psychophysiology. 1990;27(5):586-598.

35. Berntson GG, Bigger JT, Eckberg DL, et al. Heart rate variability: origins, methods, and interpretive caveats. Psychophysiology. 1997;34(6):623-648.

36. Berntson GG, Lozano DL, Chen Y-J. Filter properties of root mean square successive difference (RMSSD) for heart rate. Psychophysiology. 2005;42(2):246-252.
37. Goedhart AD, van der Sluis S, Houtveen JH, Willemsen G, de Geus EJC. Comparison of time and frequency domain measures of RSA in ambulatory recordings. Psychophysiology. 2007;44(2):203-215.

38. Park G, Van Bavel JJ, Vasey MW, Thayer JF. Cardiac vagal tone predicts inhibited attention to fearful faces. Emotion. 2012;12(6):1292-1302.

39. Nunan D, Sandercock GRH, Brodie DA. A quantitative systematic review of normal values for short-term heart rate variability in healthy adults. Pacing Clin Electrophysiol. 2010;33(11):1407-1417.

40. Wheat AL, Larkin KT. Biofeedback of heart rate variability and related physiology: a critical review. Appl Psychophysiol Biofeedback. 2010;35(3):229-242.

41. Burgess PW, Alderman N, Evans J, Emslie H, Wilson BA. The ecological validity of tests of executive function. J Int Neuropsychol Soc. 1998;4(6):547-558.

42. Chan RC. Dysexecutive symptoms among a non-clinical sample: a study with the use of the Dysexecutive Questionnaire. $\mathrm{Br} J$ Psychol. 2001;92(3):551-565.

43. Simblett SK, Bateman A. Dimensions of the Dysexecutive Questionnaire (DEX) examined using Rasch analysis. Neuropsychol Rehabil. 2011;21(1):1-25.

44. Miller MD, Paradis CF, Houck PR, et al. Rating chronic medical illness burden in geropsychiatric practice and research: application of the Cumulative Illness Rating Scale. Psychiatry Res. 1992;41(3):237-248.
Psychology Research and Behavior Management

\section{Publish your work in this journal}

Psychology Research and Behavior Management is an international, peerreviewed, open access journal focusing on the science of psychology and its application in behavior management to develop improved outcomes in the clinical, educational, sports and business arenas. Specific topics covered in the journal include: Neuroscience, memory and decision making; Behavior

\section{Dovepress}

modification and management; Clinical applications; Business and sports performance management; Social and developmental studies; Animal studies. The manuscript management system is completely online and includes a very quick and fair peer-review system, which is all easy to use. Visit http://www. dovepress.com/testimonials.php to read real quotes from published authors. 